\title{
Influence of Multisensory Stimulation Environmental Designs for Day Services and Healing Environment of Elderly People with Dementia
}

\author{
Shu-Ying Tsai* and Syuan-Yuan Hong \\ Department of Architecture \& Graduate Institute of Architecture and Urban Design, \\ National Taipei University of Technology, Taipei City 106, Taiwan, ROC.
}

(Received May 8, 2018; accepted April 23, 2019)

Keywords: healing environment, multisensory stimulation environment, day services center, elderly with dementia

The number of patients with global dementia has reached about 50 million. According to the Ministry of the Interior's July 2017 demographic data, the number of people with dementia over the age of 65 in Taiwan currently reaches 250000. The literature at home and abroad shows that multisensory helps with cognitive and behavioral improvements; therefore, 11 participants were invited to participate in the study in order to explore how the day services in Taiwan use multisensory-stimulated environments to slow cognitive deterioration and achieve physical and mental recovery. Experts include doctors, functional therapists, architects, and social workers. The following conclusions were reached: First, the targets of the multisensory stimulation environment are the intervention of nature, past life experiences, the memory and beliefs of the culture, and characteristics of Taiwan's various regions. Second, the interior design includes "fixed" and "adjustable" models and can be converted according to the needs of individuals. Third, multisensory stimulation distributed in day services are more able to respond to the care needs of different aged people than an independent sensory room.

\section{Introduction}

According to the World Health Organization (WHO) report on dementia in 2015, a dementia patient is diagnosed every $3 \mathrm{~s}$ in the world, and the population afflicted by this disease doubles every 20 years. According to the investigation by the dementia association and the estimation of the Ministry of the Interior in December 2017, there are 270000 people with dementia in Taiwan, and one in every 100 elderly persons suffers from dementia. ${ }^{(1)}$ Not only has the United Kingdom begun to apply multisensory environmental stimuli for nondrug treatment, but Spain, Australia, and the United States have also begun to apply multisensory stimulation in the care of the elderly with dementia. Because the feelings and behaviors of the elderly are different in the multisensory stimulation environment, appropriate adjustments must be made for their current moods and needs so that the elderly can experience the multisensory stimulation environment in a comfortable mood without rejection or the increase of trouble. Considering the cultural

*Corresponding author: e-mail: tsaishuying0914@gmail.com https://doi.org/10.18494/SAM.2019.2281 
differences from the Western countries, 11 participants were invited to take part in the study. Experts in the industry, government, and academia, including doctors, functional therapists, architects, and social workers, also held seminars to share their practices and experiences. An expert forum was held to elucidate the perfect environmental plan for the elderly with dementia, using data on 5-day services in Taipei with the same sensory function classroom, and responses of five characteristic design questionnaires from the literature integrating the opinions of architects, physicians, functional therapists, social workers, and interior designers. Therefore, this study is conducted (1) to better understand the concept of the multisensory stimulation environment, (2) to explore the effects of the multisensory stimulation environment on the autonomic expression and cognitive function of the elderly, and (3) to explore how the multisensory stimulation environment should be placed in the day services and to provide relevant unit references.

\section{Materials and Methods}

In this study, five multisensory stimulation environment features in day service centers were compiled from the related literature, and expert symposiums were held. Experts and scholars were asked to fill in the questionnaires before starting the open discussion of multisensory stimulation environmental issues. Exploring the way of constructing the indoor environment and drawing the most accurate discussion results for comparative analysis, we attempted to determine the most suitable construction and configuration for elderly people to be immersed in the sensory environment.

\subsection{Literature review}

According to the National Adult Day Services Association's new interpretation of adult day services, in the community-based environment, adult day service centers provide a professional integrated service program for adults with day service needs who require a safe place outside the home during the day. ${ }^{(2)}$ The establishment of such day services is designed to enable the children of elderly people to work with peace of mind, to promote interpersonal communication among the elderly to delay aging, to live enjoyably and relax, to reduce social isolation, and to enhance self-reliance. ${ }^{(3)}$ Many empirical studies have shown that a positive design for those with dementia can be assisted by a pleasant physical environmental design, and supportive spatial features, such as a direct moving line system, should be visually accessible, that is, meaningful integration should be implemented. Environmental characteristics such as lighting, sound, temperature, color, and mode are all important. ${ }^{(4,5)}$ The sensory room can make the elderly people feel positive emotions on the psychological level and can realize the importance of interpersonal friendship and environment for the elderly with dementia. ${ }^{(6)}$ Because of the involvement of sensory activities, the sensory room promotes smooth communication and rapport between the elderly and the nursing staff encourages the elderly to freely explore the sensory environment; it also promotes the most appropriate sensory activities in accordance with the current mood and physical strength. It is not mandatory to arrange fixed courses 
and time, but rather, a respect for their inner feeling is needed to achieve the most appropriate healing effect. ${ }^{(7)}$ The caregivers' involvement with sensory equipment allows the elderly to spontaneously express themselves and communicate during relaxation. In the process of interaction, the attitude of the elderly is found to change from passive participation to active participation, and the multisensory environment can be regarded as a cure improvement method. ${ }^{(8)}$ Because of the diversified settings in the sensory room, caregivers and therapists can use the environment to stimulate the five senses in accordance with different activities of elderly people. The internal space must make the elderly feel comfortable and at ease; therefore, constructing a people- or caretaker-oriented indoor environment is very important. ${ }^{(9)}$ The elderly and disabled people should have different feelings when they are in contact with the sensory room. The latter have less past experience and the sensation is surreal. Curiosity is greater than it is in the elderly. For the elderly, the contact between the sensory environment and the past experience cannot be established. Therefore, it is necessary to combine the past experiences of the elderly and the real environment to create a harmonious effect, so that they can truly enjoy the healing process of comfort and peace of mind. Before conducting activities, it is necessary to evaluate the physical condition of the elderly before in this environment, so that participation in a simulation activity involving the five senses can achieve the most effective healing process; the influences of the sensory environment not only exist in adult day services, but also extend to the community and even the family. Finally, sensory stimulation presents a continuous healing effect. $^{(10)}$ Environment and interaction are the characteristics of this multisensory room, which allows the elderly to respond positively and receive environmental support and stimulation of the body and mind. ${ }^{(11)}$ When planning the sensory room, ask caregivers and experts for suggestions on appropriate designs and how to improve the efficiency of use, and reduce the distance between the interior space and the elderly, to make the room an attractive and comfortable environment. In addition, the common problems and solutions are also mentioned in the planning manual to increase the number of practical references of actual use. ${ }^{(12)}$

The assessment of the sensory room setting is done with the following six indicators:

1. Accessibility: The environment setting inside the sensory room can be linked to reality, making it easy for the elderly to access.

2. Environmental support: The internal configuration design allows the elderly to participate in the appropriate sensory stimulation in real time and then prompts the elderly to slowly interact with other sensory equipment contents and adapt to the internal space atmosphere.

3. Diversity: The variety of equipment inside the sensory room enables the caregiver to choose the sensory stimulation that is suitable for the elderly.

4. Safety: The design of the sensory interior requires designers to work with professionals to achieve internal security.

5. Interactivity: The sensory equipment interacts with the elderly to produce a positive reflection and promote mental and physical health.

6. Autonomy: The sensory room causes the elderly to actively express themselves, resulting in positive effects on the interpersonal relationships and communication response to caregivers and peers. 
During this study, an expert symposium was held, and a questionnaire based on the literature was prepared to clarify the five characteristics of the multisensory stimulation environment. The rating of the questionnaire items is divided into four levels, strongly disagree, disagree, agree, and strongly agree, which are given scores from 1 to 4 , respectively. The expert questionnaire is detailed in Table 1.

According to the statistics of the responses to the expert questionnaire (Table 1), the scores are highest in order of safety and autonomy (37 points), environmental support (35 points), diversity (33 points), and accessibility and interactivity (31 points). It is learned from the questionnaire that the experts agree that the multisensory stimulation environmental

Table 1

Questionnaire score statistics.

\begin{tabular}{|c|c|c|c|}
\hline & & Total score & Average score \\
\hline \multirow{2}{*}{ Accessibility } & $\begin{array}{l}\text { The sensory room is positioned such that the moving } \\
\text { distance is small to avoid anxiety. }\end{array}$ & 33 & \multirow{2}{*}{31} \\
\hline & $\begin{array}{l}\text { The sensory room is close to the relevant activity field to } \\
\text { consider the demand and comfort. }\end{array}$ & 29 & \\
\hline \multirow{7}{*}{$\begin{array}{l}\text { Environmental } \\
\text { support }\end{array}$} & $\begin{array}{l}\text { The environment makes the elderly feel comfortable and at } \\
\text { ease. }\end{array}$ & 38 & \multirow{7}{*}{35} \\
\hline & $\begin{array}{l}\text { Natural light is abundant in the environment and the } \\
\text { lighting is designed with adjustable illuminance. }\end{array}$ & 38 & \\
\hline & $\begin{array}{l}\text { The sensory room has natural materials to connect with the } \\
\text { elderly. }\end{array}$ & 36 & \\
\hline & The sensory interior induces a sense of nostalgia. & 29 & \\
\hline & $\begin{array}{l}\text { The wall presents a natural scenery and the sound of } \\
\text { insects is played. }\end{array}$ & 33 & \\
\hline & $\begin{array}{l}\text { Different culture settings are considered for sensory } \\
\text { themes. }\end{array}$ & 34 & \\
\hline & $\begin{array}{l}\text { Participation activities in the sensory room can increase the } \\
\text { cognitive ability of the elderly in the short term. }\end{array}$ & 34 & \\
\hline \multirow{3}{*}{ Diversity } & $\begin{array}{l}\text { Five-sense stimulation devices work together to achieve a } \\
\text { comprehensive sensory stimulation experience. }\end{array}$ & 32 & \multirow{3}{*}{33} \\
\hline & $\begin{array}{l}\text { Facilities and abundant teaching materials promote multiple } \\
\text { choices for multisensory stimulation for the elderly. }\end{array}$ & 33 & \\
\hline & $\begin{array}{l}\text { Flexible use of this sensory device owing to the variety of } \\
\text { facilities and equipment. }\end{array}$ & 34 & \\
\hline \multirow{4}{*}{ Safety } & $\begin{array}{l}\text { Interior design must be maintained to avoid injuries caused } \\
\text { by collision. }\end{array}$ & 37 & \multirow{4}{*}{37} \\
\hline & $\begin{array}{l}\text { Sensory equipment must have documentation of factory } \\
\text { certification and follow-up maintenance. }\end{array}$ & 35 & \\
\hline & $\begin{array}{l}\text { Discussion with sensory, functional therapists and other } \\
\text { professionals to discuss sensory stimulation environment } \\
\text { configuration recommendations. }\end{array}$ & 38 & \\
\hline & $\begin{array}{l}\text { Need to cooperate with regulations to set up barrier-free } \\
\text { space that the elderly can use with peace of mind. }\end{array}$ & 36 & \\
\hline \multirow{3}{*}{ Interactivity } & $\begin{array}{l}\text { Join technology robotic pets enable the elderly to explore } \\
\text { and connect their past life experiences. }\end{array}$ & 31 & \multirow{3}{*}{31} \\
\hline & The wall design makes the elderly curious. & 34 & \\
\hline & $\begin{array}{l}\text { Smart technology devices have been designed to actively } \\
\text { sense the current needs of the elderly. }\end{array}$ & 29 & \\
\hline \multirow[b]{2}{*}{ Individuality } & The elderly is allowed to make choices independently. & 38 & \multirow[b]{2}{*}{37} \\
\hline & $\begin{array}{l}\text { The type of activity should be based on the physical and } \\
\text { mental conditions of the elderly on the day. }\end{array}$ & 35 & \\
\hline
\end{tabular}


indicator project is to be constructed in a day service, but it can be shown by the score that the multisensory stimulation must be constructed on the basis of safety and autonomy of the elderly, which is a suitable multisensory stimulation environment. Regarding environmental support, experts believe that the atmosphere of the multisensory environment makes the elderly feel comfortable. Introducing natural light and adjusting the illuminance of indoor lamps provide high familiarity to the elderly. In terms of safety, constructing a multisensory stimulation environment requires that attention be paid to sharp corners and the avoidance of collision among elderly people; thus, while planning a new sensory space, architects, nursing teachers, and functional therapists suggest that a safe configuration environment should be designed in accordance with the behavioral pattern of the elderly, emphasizing individuality. The multisensory stimulation environment allows older people to make choices independently and to have psychological support, dignity, and respect.

\subsection{Expert forum}

(1) Basic information of experts

During the study, an expert symposium was held on January 29, 2017; 11 experts from industry, academy and government were invited and requested to complete the questionnaire as well as to provide suggestions for revision on the basis of their professions. The background information of experts is given in Table 2 .

(2) Open discussion

A. Five characteristics of sensory stimulation indoor environment planning

a. Accessibility - object-linked environment

(a) A place that can be easily passed

“... accessibility is very important. In design, this can't be too secret, but there is a place that can be easily passed. We want to design the corridor in the space where

Table 2

Background information of experts.

\begin{tabular}{lccc}
\hline $\begin{array}{l}\text { Identification } \\
\text { number }\end{array}$ & Name & Affiliation & Title \\
\hline A1 & Liu & $\begin{array}{c}\text { Taipei Veterans General Hospital, } \\
\text { Attending physician }\end{array}$ & Director \\
\hline A2 & $\mathrm{Xu}$ & Red Heart Association & Deputy secretary general \\
\hline A3 & Lu & $\begin{array}{c}\text { Zhongshan Elderly Housing and } \\
\text { Service Center }\end{array}$ & Social worker \\
\hline A4 & Zheng & St. Joseph's Dementia Elderly Care Center & Occupational therapist \\
\hline A5 & Ji & Chang Gung Dementia Center & Occupational therapist \\
\hline B1 & Chen & $\begin{array}{c}\text { Department of Health, } \\
\text { Taipei City Government }\end{array}$ & Section head \\
\hline C1 & Chen & $\begin{array}{c}\text { Chung Yuan University } \\
\text { Department of Architecture (retired) }\end{array}$ & Associate professor / Architect \\
\hline C2 & Lin & Archiz C. H. Lin Architects & Architect \\
\hline C3 & Fu & CTW Design International Ltd. & General manager \\
\hline C4 & Zheng & $\begin{array}{c}\text { National Taipei University of Technology } \\
\text { Department of Industrial Design }\end{array}$ & Associate professor \\
\hline C5 & Zhang & $\begin{array}{c}\text { University of Taipei } \\
\text { Department of Health and Welfare }\end{array}$ & Assistant professor \\
\hline
\end{tabular}


we walk to emit sounds when the wall is pressed and let the elderly interact with the environment..." (A4)

(b) Nature is integrated into the original life situation

"...the design of the integration of nature and the design of the old life situation part of the elderly is the biggest help..." (C5)

b. Safety - reduce the chance of injury for the elderly

(a) Turn into a barrier-free heart

"...The four levels are accessible, versatile, then friendly, and finally healed. Accessibility is the basis of an available environment. Versatility is created by adding a few more objects, and when the environment is friendly, it has changed from physically barrier-free to psychologically barrier-free..." (C1)

(b) Furniture is as simple as possible

"...the big space includes walls, because for patients with dementia, when things are complicated, or we feel that sensory equipment is very rich, the elderly will feel more confused..." (A5)

c. Interactivity - establish a microsocial model and increase the frequency of communication

(a) Communication between people

“...first, enhancing one's activities of daily living [ADL], also creates a healing environment... sensory equipment is the communication between people based on social models, and the communication between people and groups..." $(\mathrm{C} 1)$

(b) Become a great listener

"...The doctor is getting dementia by himself, but he still remembers his own occupation; he is sitting in that place and has become a very good listener. There will be someone talking to him over there..." (C5)

d. Autonomy - guide elderly people to choose the appropriate sensory stimulus

(a) Design specific path

"... and then to the needs of the individual elderly, the daily routine is his own choice and is related to the purpose of the sensory environment corner, therefore, this space has a specific path within the trip. With this specific corner, the behavior makes him independent and individualized..." (C2)

(a) Very interested in this cooking activity

“...the world of food involves interaction with food. Because nearly 70\%...75\% of the elders are women, and housewives are very interested in this food event..." (A3)

e. Gameplay - Inspire curiosity in the elderly and allow the elderly to intuitively manipulate the content of the activity

(b) Make him curious

"...First, you have to make him curious... you may have to add gameplay, otherwise the elderly would not be interested in playing with you there..." (C4)

(c) She will forget her black dog when she dances with others

"...with a Wii, she can be there and wave... that can improve her ADL... and relieve some degree of psychological stress. She can sing with people, and dance with 
others, then she will forget her black dog, which is a cause of her depression..."(C1)

B. Leading the elderly to the sensory room environment and the difficulties encountered and their solutions

Stimulating environment for construction-furniture equipped with movable furniture to create flexibly adjustable space

(a) There will be fewer fixed items and more movable components

“...It's not that these things are all designed by us as a fixed and single design; but that this space can be adapted to a lot of applications. These devices are mobile and can be moved in and out. Some of the most basic elements of this space are a "changeable" variety of types. This multisensory environment will have fewer fixed items in this class and more movable components..." (A1)

(b) The background of life experiences and seasonal adjustments at that time

“...We have a simple space, but the materials can be adjusted at any time regardless of the age of the patient, or the background of life experiences and the seasonal adjustments at that time..." (A5)

C. Give appropriate sensory stimulation to elderly people exhibiting different ailments

Different illnesses are given to different activities to design - to carry out activities

(a) Be empathic

“...The last question is to put yourself in the shoes of others... Today we are here for some people with limited mobility or other impairments. In a garden, some visually impaired people say that they usually have no chance to touch the plants, even the elderly have much more interaction there..." (C5)

(b) Understand the life history of these subjects

"...The first element may be what you know about him to make him very comfortable in this environment... mild cognitive impairment [MCI] is more suitable. When medium to severe CDR is relatively high, in fact, his BPSD is related to the environment..." (C1)

D. The sensory stimulation environment needs to be dispersed or concentrated.

a. Spread throughout the center - the elderly can choose the most suitable sensory environment for the moment

(a) Sensory environment scattered into various spaces and active in public spaces

"...scattered to various spatial planning ideas, mainly to allow the elderly to walk in public spaces, walking in the forest trails combined with Wenshan, Jingmeixi and Jingmei Night Market, and playing natural music to feel the auditory stimulation..." (A2)

(b) The concentrated sensory room will cause a heavy burden

“...It should be scattered, because I feel that a centralized sensory room will cause a heavy burden. In particular, some equipment will be difficult to install at first... but I feel that if it is dispersed, many ideas will come of it..." (A1)

b. Concentrate one setting

(a) It is a warm feeling

"...When the center was redesigned, it raised a warm feeling. There were some 
natural plants in it, so it was emphasized that this Snoezelen room mode is suitable for all ages, and for all abilities..." (A4)

(b) Space interaction

"...If the space is large enough to set up a sensory classroom, basically, in terms of day services, we don't just have sensory activities in the sensory classroom. We must use it alternately, including cognitive activities and also art activities..." (A3)

E. The necessity of sensory room setting and the length of time to improve cognitive function and behavioral performance of elderly people

a. Construction based on building space - flexible use of space

(a) Make good use of technology

"...we want to be able to make good use of these technologies. When you are alone, you can use virtual sounds for stimulation, including air control; but you have to connect with others when you are not alone..." (C4)

(b) Have a relaxing space

“...no need to set up a sensory room... From the perspective of sensory stimulation, I think it is important to have a space for relaxation..." (A4)

b. Sensory stimulation alleviates symptoms of elderly patients' illness

(a) Continuous contact

"...I think it should enhance these cognitive abilities, so it is necessary to continue contact. Maybe a normal activity offered by this institution could achieve it..." (A1)

(b) Personal life needs to be integrated into the situation

"...the situation should be integrated with his personal life need; this is the reason why situation design is important..." (C5)

F. Develop regulations for the benefit of sensory stimulation of the environment

\section{Legal requirements should be the minimum standard}

(a) Think of $6.6 \mathrm{~m}^{2}$ as a minimum standard

"... Be able to see $6.6 \mathrm{~m}^{2}$ as a minimum standard, so that there is a chance to create more space..." (C2)

(b) The size of the space is irrelevant

"...The size of the space is irrelevant. If my space is small, then I might simply use a simulation room set..." (C4)

G. Doubts about five sensory stimuli and simultaneous intervention

\section{Proper stimulation}

(a) The senses should be appropriate

"...in terms of the concentration of dementia patients and the characteristics of their irritability, the senses should be appropriate..." (A1)

(b) The five senses are distributed in different spaces and life scenes

"...The five senses are distributed in different spaces and combined with life... In fact, they do not exist together in one place. Vision may be here, hearing over there..." (C1)

H. Establish a comfortable multisensory environment in day services

Multisensory stimulating environment with regional characteristics 
(a) Fusion landmarks

"....Our fusion structure is just the Dazhi Bridge in the Zhong-Shan area, it is built with a water pipe light. The light will be lit up by the sounds from a speaker. The louder the sound, the brighter the light will be..." (A3)

(b) Make a natural scene on the wall

“...To make a birds'-eye view natural scenery on the wall, or to give specific auditory and visual stimulation, and then provide specific training activities, you can achieve this purpose..." (A5)

(3) Summary

On the basis of the sensory characteristics provided by the experts, in addition to the accessibility, security, interactivity, and personality in the literature, the experts also increase autonomy, gameplay, fun and learning. In terms of space planning, there are still experts' suggestions. The modular indoor space pattern must be constructed, and the fixed and movable furniture can be used to make the activity space flexible. The internal structure of the space changes in accordance with the dementia. In addition, the sensory room and sensory stimulation environment have their own advantages and disadvantages. Some experts believe that sensory stimulation should be scattered throughout the space of day services, and the elderly can receive appropriate stimulation depending on their condition that day. Other experts agree with this method, but also recommend providing the public with some explanation for the closed sensory room, the many shiny devices inside, and the internal cool tones and atmosphere. For example, the internal color tone and indoor layout can be adjusted to reflect the era of objects and provide a homey feeling. Otherwise, adding some plants can soften the stiffness of the space and provide a sense of psychological ease. Experts also point out that the sense stimulations inside day services can incorporate nature and regional cultural characteristics, connecting the elderly with their life style and presenting the most appropriate healing environment. From a barrierfree environment to a healing environment, the inner body, mind and spirit of the elderly are cured. The connection with life experience is further designed, plus the elderly has the right to choose independently, to achieve a dignified old living environment.

\section{Discussion and Analysis}

Experts believe that multisensory environmental characteristics can alleviate the symptoms of the elderly, and pointed out that one can add gameplay and learning; therefore, sensory stimulation is not merely a feeling, but also allows elderly people to learn new things and induce, in themselves, a sense of accomplishment. However, the five indicators and total scores presented in the questionnaire indicate that the experts believe that the most important indicators are safety and personality. In addition, in open discussions, safety is also reflected in the construction of indoor planning and design as one of the most basic concepts, and it is recommended that the space configuration be further discussed with relevant experts in the planning process, to better recognize the needs of the elderly and caregivers. Currently, the regulations comprise only minimum thresholds, and hence, high standards should be designed by architects and interior designers. In terms of individuality, the elderly should be given the 
autonomy to choose the sensory environmental stimuli they need, and the appropriate sensory stimulation is managed by the caregiver, and the growth background of each senior citizen and different sensory stimuli are given. A customized sensory course experience maximizes the alertness and awareness of the elderly. The literature mentions that comfortable sensory environmental stimuli can calm the cognitive function in elderly people, ${ }^{(8)}$ but experts believe that transient sensory environmental stimuli are insufficient to maintain the cognitive function and suggest that continuous stimulatory intervention is needed to be effective for elderly people.

\section{Conclusions}

The care environment for people with dementia should be based on a people-oriented approach when considering design, and the lifestyles that elderly people are accustomed to should be extended to the care unit to reduce the internal pressure caused by the care environment. In an insufficient space, there is a better way of arranging the sensory room to incorporate the atmosphere of the home and to reduce the anxiety of the elderly entering into a strange space so as to achieve the healing effect by enticing the elderly to undergo sensory stimulation. Thus, there needs to be an intermediary space for conversion, such as a dynamic, static, artificial, or natural space. Each person of senior age can choose multisensory stimulation in accordance with his personal preference, so that he can gain peace of mind and comfort on the psychological level, and reduce pressure of entering into unfamiliar environments and interpersonal relationships through appropriate sensory stimulation. Excessive sensory stimulation is easy to emotionally disturb and incite. Continuous and appropriate sensory stimulation helps improve the cognitive ability of the elderly and reduce depression. Therefore, experts believe two stimulations per day to be sufficient. The sensory stimulation environment is not only in day services, but also in the community and even in the elderly's own home. A healing and unique environment that indirectly triggers in the elderly motivation to actively share and increase the frequency of interpersonal interactions should be established. The cultural characteristics of the locality should be incorporated into day services, along with familiar Taiwanese culture such as night market vendors, so that the elderly can directly experience the sensory stimulation. It is recommended that when a sensory environment stimulus is established in the future, it can be extended from day services to the community and family to induce continuous sensory stimulation. The conclusions are summarized as follows.

1. The standard size of a space stipulated by the regulations is not the size of a comfortable space, so experts recommend that the regulations be the minimum standards and that architects and interior designers incorporate higher standards.

2. When accessibility is raised to the healing environment, indoor spatial planning should not only be physiologically safe for the elderly, but also promote a psychologically stable existence.

3. The day services in the process of being constructed, because of two insufficient indoor spaces used to plan a large sensory stimulation, make good use of technology in combination with a large number of storage cabinets and movable furniture to activate the space. 


\section{Acknowledgments}

We would like to thank the Ministry of Science and Technology for providing research funding for "The interaction of the elderly and the environment in the Rizhao Center with the multisensory environment design" (No. MOST 106-2410-H-027-012).

\section{References}

1 Taiwan Alzheimer Disease Association: http://www.tada2002.org.tw/ (accessed April 2018).

2 E. G. Joseph: Home Health Care Serv Q. 33 (2015) 1.

3 J. Anke and C. Lesley: Design 4 Health 3 (2015) 13.

4 R. Fleming and N. Puarn dare: Int. Psychogeriatr. 22 (2010) 7.

5 G Marquardt, K. Bueter, and T. Motzek: HERD 8 (2014) 1.

6 J. J. Lopez, J. C. Bolivar, and M. S. Perez: Article in Dementia 15 (2014) 4.

7 B. Sarah, V. D. Erik, and P. Richard: Adv. Psychiatr. Treatment 8 (2002) 444.

8 K. W. Hope, R. Easby, and H. Waterman: J. Psychiatry Ment. Health Nurs. 11 (2004) 5.

9 K. W. Hope: J. Psychiatry Ment. Health Nurs. 5 (1998) 5.

10 Y. Cui, M. Shen, Y. Ma, and S. W. Wen: Med. Hypotheses 98 (2017) 11-14.

11 S. Behrman, L. Chouliaras, and K. P. Ebmeier: Maturitas 77 (2014) 4.

12 Ministry of Health and Welfare: https://www.mohw.gov.tw/ (accessed April 2018).

\section{About the Authors}

Shu-Ying Tsai received her B.S. and M.S. degrees from the Department of Architecture, National Cheng Kung University, Taiwan, in 1980 and 1982, respectively, and her Ph.D. degree from the Graduate Institute of Building \& Planning, National Taiwan University, Taiwan, in 2001. She is an associate professor at National Taipei University of Technology. Her research interests are in geronarchitecture and human and environment research.

Syuan-Yuan Hong received his B.S. degree from Shu-Te University, Taiwan, in 2010. Since 2016, he has been a research assistant at National Taipei University of Technology. 